\title{
¿INFLUYE EL SEXO EN EL RETRASO DE LA VINCULACIÓN AL PROGRAMA TARV EN LOS PACIENTES CON VIH?
}

\author{
DOES SEX INFLUENCE IN THE DELAY OF LINKAGE TO HAART IN HIV PATIENTS? \\ Fiorella Arce-Huaroto ${ }^{1, a}$, Angela Villanueva-Del-Busto $1, a$
}

\begin{abstract}
Sr. Editor
La epidemia de VIH representa un problema de salud pública a nivel mundial, afecta 36,9 millones de personas en el mundo y se estima que causa más de un millón de muertes por año. En el Perú, para el mes de diciembre del 2018, según el reporte de la Dirección General de Epidemiologia se notificaron un total de 120389 casos de infección por VIH y de los cuales 43072 se encontraban en estadio SIDA. ${ }^{1}$

El objetivo de la terapia antirretroviral (TARV) anteriormente reconocida como terapia antirretroviral de gran actividad (TARGA) es suprimir el virus hasta niveles indetectables de modo que se pueda preservar o restaurar la función inmune, logrando disminuir la mortalidad y mejorar la calidad de vida. Sin embargo, según la OMS, hasta el año 2017, solo 67\% de las personas infectadas recibían TARV². La demora en la vinculación a dicho programa en pacientes con diagnostico positivo de infección por VIH contribuye al riesgo de propagación de la enfermedad, el deterioro inmunológico, aumento de la morbilidad, así como mayores gastos directos de tratamiento médico estimándose que los gastos acumulativos aumentan cuanto más tiempo demore un paciente en iniciar tratamiento.
\end{abstract}

Diversos estudios hallaron resultados discordantes en cuanto al sexo masculino y su significancia para el inicio tardío de la terapia antirretroviral (Tabla 1). Parrott et $\mathrm{al}^{3}$, Van der Kop et $\mathrm{al}^{4}$, Nedezuko et $\mathrm{al}^{5}$, obtuvieron en sus estudios que el sexo masculino constituía un factor de riesgo para el inicio tardío de la terapia antirretroviral. Los hombres tendrían la percepción que el iniciar TARV afectara su masculinidad, se aumentaría la visibilidad de su estado de $\mathrm{VIH}$ y los pondría en riesgo de estigma externo como el rechazo de sus parejas actuales, compañeros, familiares y miembros de la comunidad. Además, se señala que los hombres generalmente suelen minimizar sus malestares, resguardarse en el hecho de no sentirse enfermos, restando importancia al inicio temprano de la terapia antirretroviral, ${ }^{5}$ Por otro lado, Turner et al ${ }^{6}$ no hallaron asociación.

Debido a la discrepancia de los resultados en cuanto al sexo masculino como factor de riesgo para el inicio tardío de la terapia antirretroviral y que los estudios mencionados fueron realizados en otras regiones (Kenia, Ucrania, Malawi y Estados Unidos), se sugiere realizar estudios locales puesto que se considera importante reconocer si el sexo masculino constituye un factor de riesgo porque se evidencia que dicho grupo tiene una mayor prevalencia de infección por VIH. Según el Centro de Epidemiologia, Prevención y Control de Enfermedades, en Perú hasta diciembre del 2018 la relación según el sexo fue de 3,7 hombres por una mujer en los casos diagnosticados de infección VIH y para los casos SIDA 4,5 hombres por una mujer'; así como, en un estudio realizado en un hospital en Lima se encontró que el sexo masculino tuvo $17 \%$ más frecuencia de diagnóstico tardío comparado con las mujeres y nos podría indicar que los hombres demoran más en acudir a los centros de salud en busca de atención ${ }^{6}$, lo cual podría implicar un retraso de la vinculación al programa de terapia antirretroviral de gran actividad. Es pertinente mencionar que otros aspectos pueden jugar un rol importante en el retraso en la vinculación al programa TARV, como por ejemplo la estructuración del sistema de salud, los hombres al ser una población económicamente activa tienden a priorizar sus actividades laborales que sumado a un deficiente sistema de salud pueden conllevar al retraso en la búsqueda y consiguiente vinculación al programa TARV.

1 Facultad de Medicina Humana, Universidad Ricardo Palma, Lima-Perú.

Estudiante de Medicina.

Citar como: Fiorella Arce-Huaroto, Angela Villanueva-Del-Busto. ¿Influye el sexo en el retraso de la vinculación al programa TARV en los pacientes con VIH?. Rev. Fac. Med. Hum. Octubre 2019; 19(4):131-132. DOI 10.25176/RFMH.v19i4.2196 
La identificación de los factores sociodemográficos que influyen en las decisiones de las personas con infección por VIH para buscar atención e iniciar un tratamiento, permitirá el diseño de intervenciones efectivas de salud pública para aumentar el inicio temprano de la terapia antirretroviral en pacientes con diagnóstico de infección por VIH.

Tabla 1. Influencia del sexo masculino en inicio tardío del programa TARV según estudios previamente publicados.

\begin{tabular}{|c|c|c|c|c|}
\hline Autores & Muestra & País & Tipo de estudio & $\begin{array}{c}\text { Efecto del sexo masculino en } \\
\text { inicio tardío }\end{array}$ \\
\hline Turner et al. & 3500 & Estados Unidos & Cohorte & $\mathrm{OR}=0,98(\mathrm{IC} 95 \% 0,56-1,72)$ \\
\hline Parrott et al. & 365 & Malawi & Cohorte & $\mathrm{OR}=2,30(\mathrm{IC} 95 \% 1,30-3,90)$ \\
\hline Van der Kop et al. & 775 & Kenia & Transversal & $\mathrm{OR}=1,30(\mathrm{IC} 95 \% 0,93-1,82)$ \\
\hline Nedezhuko et al & 200 & Ucrania & Transversal & $\mathrm{OR}=1,2(\mathrm{IC} 95 \% 0,69-2,1)$ \\
\hline
\end{tabular}

Contribuciones de autoría: los autores participaron en la generación, recolección de información, redacción y versión final del artículo original.

Financiamiento: Autofinanciado.

\section{Correspondencia: Fiorella Arce-Huaroto}

Dirección: Julio C Tello 220 Urb San José, Bellavista - Callao, Lima-Perú

Teléfono: 989-202859

Correo: fiorella.arce@urp.edu.pe
Conflicto de interés: Los autores declaran no tener conflictos de interés en la publicación de este artículo.

Recibido: 21 de julio del 2019

Aprobado: 11 de septiembre del 2019

\section{REFERENCIAS BIBLIOGRÁFICAS}

1. Centro Nacional de Epidemiologia, Prevención y Control de Enfermedades [Internet]. [citado el 16 de mayo de 2019]. Disponible en:https://www.dge.gob.pe/portal/index.php?option=com content\&view=article\&id=656

2. GHO|By category |Antiretroviral therapy coverage - Data and estimates by WHO region [Internet]. WHO. [citado el 16 de mayo de 2019]. Disponible en: http://apps.who.int/gho/data/view.main.23300REGION?lang=en

3. Parrott FR, Mwafulirwa C, Ngwira B, Nkhwazi S, Floyd S, Houben RMGJ, et al. Combining Qualitative and Quantitative Evidence to Determine Factors Leading to Late Presentation for Antiretroviral Therapy in Malawi. Myer L, editor. PLoS ONE. 2011;6(11). Disponible en: https://www.ncbi. nlm.nih.gov/pubmed/22114727.

4. Van der Kop ML, Thabane L, Awiti PO, Muhula S, Kyomuhangi LB, Lester RT, et al. Advanced HIV disease at presentation to care in Nairobi, Kenya: late diagnosis or delayed linkage to care? - a cross-sectional study. BMC Infect Dis. Dic 2016;16(1):169. Disponible en: https://www.ncbi.nlm.nih. gov/pmc/articles/PMC4835937/

5. Neduzhko O, Postnov O, Perehinets I, DeHovitz J, Joseph M, Odegaard $D$, et al. Factors Associated with Delayed Enrollment in HIV Medical Care among HIV-Positive Individuals in Odessa Region, Ukraine. Journal of the International Association of Providers of AIDS Care (JIAPAC). Marz 2017;16(2):168-73. Disponible en: https://www.ncbi.nlm.nih.gov/pmc/ articles/PMC5363501/

6. Turner BJ. Delayed Medical Care After Diagnosis in a US National Probability Sample of Persons Infected With Human Immunodeficiency Virus. Arch Intern Med. 2000;160(17):2614. Disponible en: https://www. ncbi.nlm.nih.gov/pubmed/1099997 